https://helda.helsinki.fi

\title{
The cell biology of lipid droplets : More than just a phase
}

\section{Klemm, Robin W.}

2020-12

Klemm , R W \& Ikonen , E 2020 , ' The cell biology of lipid droplets : More than just a phase '

, Seminars in Cell and Developmental Biology , vol. 108 , pp. 1-3 . https://doi.org/10.1016/j.semcdb.2020.06.016

http://hdl.handle.net/10138/337182

https://doi.org/10.1016/j.semcdb.2020.06.016

cc_by_nc_nd

acceptedVersion

Downloaded from Helda, University of Helsinki institutional repository.

This is an electronic reprint of the original article.

This reprint may differ from the original in pagination and typographic detail.

Please cite the original version. 
Editorial

\section{The cell biology of lipid droplets: More than just a phase}

Fats and oils are the most energy dense nutrient stores in living organisms. The hydrocarbon chains of biological fats are not soluble in water and separate spontaneously from the aqueous cytoplasm to form distinct phases. While the low volumes that are associated with essentially water free storage are a great advantage they come at a price, because most biochemical mechanisms operating in living cells have evolved to function in an aqueous milieu, and are not readily applicable to hydrophobic molecules. The generation and controlled maintenance of cellular compartments storing fat are, however, of central importance to many cell biological processes and vital for whole-body metabolic health. The fascinating molecular solutions that evolved to meet the mechanistic challenges of biology in the fat phase are the focus of this special review issue.

The compartmentalization of eukaryotic cells into organelles with specialized biochemical functions is one of the most fundamental principles in biology [1]. Classical organelles, such as the endoplasmic reticulum (ER), Golgi apparatus and mitochondria are surrounded by one or two phospholipid bilayers separating an aqueous lumen from the surrounding cytosol. Membrane-less organelles (MLOs) are formed in the cytoplasm by local de-mixing of proteins and RNA into dynamic assemblies, exhibiting properties of distinct liquid phases [2-4]. Lipid droplets (LDs) represent a third type of organelle containing a hydrophobic, neutral lipid filled core surrounded by a phospholipid monolayer [5].

LDs were described as early as in the late 19th century [6-8]. Initially referred to as microsomes or liposomes, they were mistakenly considered as inert, long-term lipid stores only present in specialized cells of the adipose tissue, steroid hormone glands or the liver [5]. Although evidence for their presence in a wide range of cell types steadily accumulated [9], the community largely ignored "the cellular lipid inclusions" over the next 100 years. It took until the early 1990s to re-kindle the interest in this fascinating organelle [10], with a number of pioneering labs laying out the first cell biological and molecular principles of LD biology [11-13]. Despite rapid progress and a first wave of important discoveries, LDs were not generally accepted as independent organelles. A widely spread misconception was that LDs were mere appendages of the ER membrane, where they are formed, and only responded passively to the metabolic needs of the cell.

An important breakthrough was the realization that LDs are, in fact, ubiquitously present and of general relevance for a number of cell biological and physiological functions. It was slowly recognized that virtually all cells of the human body are able to generate LDs, and the ability to build these organelles depends on evolutionarily conserved machinery. Indeed, LDs are found, with some variations, across all phyla [5]. It was, now, necessary to agree on unified terminology and the field converged on defining the "lipid droplet" [5,14]. The molecular understanding of LD biogenesis and turn-over rapidly increased
[15-23] and paved the way to the recognition of LDs as truly independent cellular organelles in the early 2000s [14,24-27].

Now, another 20 years later, the LD has come of age and has given rise to a vibrant and rapidly advancing research field [28-30]. This special review issue provides an overview of the current state of knowledge and concepts in the field by leading experts, covering a broad range of topics from the molecular basis and biophysics of LD biogenesis, to insights into sparsely charted areas in lipid and energy metabolism, and extending to the diverse functions of LDs in metabolic homeostasis across various tissues in the human body.

The first review of the special issue by Bianca Schrul, Rachid Thiam and colleagues introduces the general blue print of LD architecture, and presents an in-depth analysis of the molecular mechanisms of LD biogenesis. Arguably, the most distinguishing feature of LDs is their formation within the ER bilayer, starting from nanometer sized lens-like inclusions made of neutral lipids, such as triacylglycerols (TAGs) and sterol esters (SEs). These lipids possess different biophysical properties than membrane forming lipids and segregate from other membrane components, eventually bulging out into the cytoplasm to form a LD. One might be tempted to think that this process occurs spontaneously, governed exclusively by principles of emulsion physics, but a staggering number of proteins have evolved to regulate LD formation. The article explains the biophysical principles that operate during LD biogenesis and discusses how proteins overcome and exploit the properties of this special organelle to organize its cell biology.

The following two reviews from the labs of Pedro Carvalho and Maria Bohnert focus on the molecular basis of spatial regulation during LD biogenesis. One of the key proteins in LD biology called seipin helps to concentrate protein machinery to the LD forming sites, providing control over the positioning of LD exit sites along the ER membranes. Carvalho et.al further discuss the role of special membrane lipids in LD budding, and how seipin with its interactors integrates lipid metabolic flux during LD biogenesis. Maria Bohnert elaborates on how the seipin complex engages different components during the LD life cycle, and describes the mechanistic importance of LD interactions with other organelles. Both reviews provide a comprehensive summary on the recent advances and novel factors that control LD formation at specific organelle domains and point out their differential roles during various metabolic conditions of the cell.

In their review, Marta Bosch, Rob Parton and Albert Pol discuss how LDs and mitochondria co-operate at the center stage of metabolism by integrating a comprehensive network of energy and lipid metabolic fluxes in a tissue specific manner. Bosch et al. review the flexibility of nutrient metabolism during feeding and fasting states, with metabolic switches embedded in structural adaptations between organelles. Specifically, the authors discuss for instance the "metabolic synapse" between LDs and mitochondria and how it changes according to 
metabolic state, being relevant both during nutrient oxidation and anabolic metabolism. Moreover, the review highlights the mobility of LDs in response to changing nutrient conditions, where the microtubule cytoskeleton plays a central role, and how changes in subcellular localization can lead to functional contacts between various organelles. Especially organelles like the LD that are not part of the vesicular transport system, might critically depend on physical interactions with other organelles.

Toyoshi Fujimoto with colleagues continue on the topic of LDs in metabolic control by focusing on the connections between LDs and autophagy. Autophagy is a nutrient stress response during which cells resolve a prolonged scarcity of external food by "digesting" dispensable cellular content, including LDs. The pathway controlling the autophagic consumption of LDs is called lipophagy. Specific machineries govern several delivery routes that move LDs into the lysosome for lipid breakdown and fatty acid mobilization. These pathways are probably best understood in yeast, and select components that were introduced earlier in this issue during discussions on seipin appear to be involved. Fujimoto et al. illustrate how LDs are intricately connected to autophagy in several ways: LDs serve as a source for membrane expansion during build-up of the autophagic compartments, they are used to store fatty acids freed during autophagic degradation of other organelles, and connect to mitochondria to deliver fatty acids to beta oxidation and ATP production. The interplay between autophagosomes and LDs is extremely complex, since both organelles are highly plastic in responding to changes in cellular metabolism.

The notion of LD functional diversity is echoed in the following articles. Brown adipocytes, for instance, uncouple nutrient breakdown from ATP synthesis and instead produce heat. Orian Shirihai and coworkers review the emerging mechanisms governing the concerted interplay between LDs and mitochondria in this context. One of the most unexpected discoveries is that mitochondria forming close physical contacts with LDs seem dedicated to anabolic reactions rather than fatty acid consumption. In contrast, mitochondria not in direct contact with LDs may be more efficient in nutrient oxidation and thermogenesis. Apparently, more than one mechanism is responsible for the regulation of adaptive LD-mitochondria contact site formation. In white adipocytes, mitochondria establish contact with LDs as well, but use other LD tethering proteins than brown adipocytes. The molecular basis controlling the structural relationship between LDs and other organelles in adipocytes during catabolic and anabolic metabolism is largely unresolved, but emerges as a powerful mode of regulation.

Martina Wallace and Christian Metallo discuss the molecular pathways of de novo lipogenesis (DNL), i.e. the condensation of acyl units to synthesize fatty acids (FAs), which can then be diverted into LDs for storage as TAG. The article provides a concise review of the known fatty acid synthase (FASN) machinery, and in addition highlights the unexpected and intriguing diversity of FAs produced in various tissues. Apart from the canonical even chain FAs, DNL gives rise to a several atypical FAs, e.g. with an odd number of C-atoms, or the presence of methyl or ethyl branches throughout the acyl chain. The functions of branched chain FAs are not well understood. Importantly, Wallace et al. outline how technological advances, such as mass spectrometry based lipidomics in combination with isotope labeling, will permit better analysis of structural lipid diversity and lead to an improved understanding of their roles in cell biology and metabolic diseases.

Natalie Krahmer and colleagues provide a comprehensive review on hepatic LD homeostasis and the complex regulation of lipid metabolic pathways, organelle communication and organ-level cross-talk in human fatty liver disease. They discuss how TAG and phospholipid homeostasis are co-regulated in hepatocytes and how imbalances between lipid storage, degradation and lipoprotein secretion can lead to fatty liver disease. Dietary nutrient uptake is considered in relation to storage in adipose tissue and processing in the liver, and the role of insulin resistance in disease development is discussed. Krahmer et al. also illustrate the close structural and functional relationship between LDs and blood lipoproteins that represent a readily accessible wholebody lipid diagnostic readout. The overview on the cell biological pathways dysregulated in pathological states, e.g. during fatty liver disease and disorders of the adipose tissue as well as the metabolic malfunctions that can cause type II diabetes, is an outstanding reference for a number of pressing aims to be addressed in molecular metabolism.

Last but not least, the issue offers an extensive review of LD biology in plants and photosynthetic unicellular organisms, such as green algae. Till Ischebeck and his colleagues Robert Mullen, John Dyer, and Kent Chapman present a comprehensive summary of the molecular mechanisms generating plant LDs and describe plant oil production and consumption in different contexts. Interestingly, research in plants was at the forefront of the early molecular discoveries made in the field of LD biology. Most of the machinery relevant in mammalian LD biogenesis is conserved in plants but plants are also able to carry out metabolic functions that are not found in animals. The consumption of seed oils during germination is a prominent example. Plants break down fatty acids mobilized from TAGs in LDs by peroxisomal beta oxidation and shunt the resulting products into the tricarboxylic acid (TCA) cycle in mitochondria. One of the TCA pathway intermediates, succinate, is converted into sugars, allowing plants to grow independently of carbohydrates. Animals cannot use the products of beta oxidation without dietary carbohydrates and are therefore not able to grow on pure oil- based diets.

The reviews in this issue highlight the flourishing status of LD research and point to future directions of investigation. The diverse molecular mechanisms governing integration of cell biological and metabolic functions as well as the undisputed relevance of LDs in a wide range of human medical conditions, including metabolic disorders, cancer, virus infection and neurological diseases, will attract an everincreasing number of researchers to the field. The compositional information of the plasma "lipotype" holds promise as an integrative measure of metabolic health [31]. It is therefore critical to gain a more detailed understanding of the complex cellular feeder and consumption pathways giving rise to lipoprotein composition. Moreover, plant LDs are experiencing a major increase in attention. Green algae are a promising source for the ecological production of essential dietary fatty acids and sustainable synthesis of biofuels. Plant LD biology is also central for synthetic improvements of oil seedlings and better understanding of photosynthetic metabolism in agricultural crops that will aid in improving the food supply of the rapidly growing world population.

Preface from the Guest Editor

Robin W. Klemm ${ }^{1, *}$

1 Department of Physiology, Anatomy and Genetics, University of Oxford,

Sherrington Building, Parks Road, Oxford OX1 3PT, UK

*Guest editor: robin.klemm@dpag.ox.ac.uk

Lipid droplets (LDs) have had an arguably eventful history. Starting out almost completely ignored if not entirely dismissed as bona fide cellular compartments they are now the focus of one of the most actively researched fields in organelle biology. The basic structure of LDs with a hydrophobic core and a surface composed of a phospholipid monolayer and specialized proteins distinguishes them architecturally from other organelles, and requires an arsenal of unique mechanisms facilitating LD biogenesis and consumption.

Protein targeting to the LD from the membranes of the endoplasmic reticulum (ER) or the cytoplasm depends on specific membrane anchors that are compatible with both, the special physicochemical properties of the LD surface and lipid bilayers of conventional membrane bound organelles. The reader of this special issue does not need to be convinced how fascinating the molecular solutions are that exist to meet these mechanistic challenges, but the level of sophistication found in the LD 
machinery is even to the expert at times surprising and truly captivating. This special issue further highlights the fact that many components of the enzymatic machinery necessary to form LDs are conserved across most phyla, and programs organizing the cell biology of LDs are remarkably similar between evolutionarily distant organisms such as photosynthetic microorganisms and humans.

A theme that pervades all articles in this issue is how protein machineries act together with lipids to synthesize and turn over LDs in response to metabolic stimuli in a well-regulated, and controlled fashion. The discussion further centres on how LDs engage several other organelles in the cell to fulfil their complex tasks in energy, lipid and nutrient metabolism, and the importance of metabolic flexibility and structural plasticity of LDs for whole body metabolic health is highlighted.

An astonishing feature of LDs is their versatility in function depending on the cell type or the metabolic state of the respective tissue. In an age when obesity, type 2 diabetes and cardiovascular disease are among the biggest medical and socio-economic challenges, a thorough molecular understanding of LD biology, and its metabolic functions is more vital than ever. This special issue provides a comprehensive summary of the current state of the field, gives extensive insight into experimental approaches used in the community and sketches out future directions and important questions that need to be answered so that we can harness the enormous diagnostic power of metabolomic data, improve our medical tools, and move large sections of society to a healthier future.

\section{References}

[1] P. Mazzarello, A unifying concept: the history of cell theory, Nat. Cell Biol. 1 (1999) E13-E15.

[2] A.A. Hyman, K. Simons, Cell biology. Beyond oil and water-phase transitions in cells, Science 337 (2012) 1047-1049.

[3] S.F. Banani, H.O. Lee, A.A. Hyman, M.K. Rosen, Biomolecular condensates: organizers of cellular biochemistry, Nat. Rev. Mol. Cell Biol. 18 (2017) 285-298.

[4] Y. Shin, C.P. Brangwynne, Liquid phase condensation in cell physiology and disease, Science 357 (2017) eaaf4382.

[5] D.J. Murphy, The dynamic roles of intracellular lipid droplets: from archaea to mammals, Protoplasma 249 (2012) 541-585.

[6] R. Altmann, Die Elementarorganismen und ihre Beziehungen zu den Zellen, (1894).

[7] J. von Hanstein, Ueber die Gestaltungsvorgange in den Zellkerne bei der Theilung der Zellen, Bot. Abhandl. Morphol. Physiol. Bonn. 4 (2) (1880).

[8] D.J. Murphy, The biogenesis and functions of lipid bodies in animals, plants and microorganisms, Prog. Lipid Res. 40 (2001) 325-438.

[9] D.W. Fawcette, The Cell, 2nd edition, Saunders, 1981.

[10] D.J. Murphy, J. Vance, Mechanisms of lipid-body formation, Trends Biochem. Sci. 24 (1999) 109-115.

[11] A.S. Greenberg, et al., Perilipin, a major hormonally regulated adipocyte-specific phosphoprotein associated with the periphery of lipid storage droplets, J. Biol. Chem. 266 (1991) 11341-11346.

[12] E.J. Blanchette-Mackie, et al., Perilipin is located on the surface layer of intracellular lipid droplets in adipocytes, J. Lipid Res. 36 (1995) 1211-1226.

[13] D.L. Brasaemle, T. Barber, A.R. Kimmel, C. Londos, Post-translational regulation of perilipin expression. Stabilization by stored intracellular neutral lipids, J. Biol. Chem. 272 (1997) 9378-9387.

[14] S. Martin, R.G. Parton, Lipid droplets: a unified view of a dynamic organelle, Nat. Rev. Mol. Cell Biol. 7 (2006) 373-378.

[15] R.A. Coleman, D.P. Lee, Enzymes of triacylglycerol synthesis and their regulation, Prog. Lipid Res. 43 (2004) 134-176.

[16] S. Cases, et al., Identification of a gene encoding an acyl CoA:diacylglycerol acyltransferase, a key enzyme in triacylglycerol synthesis, Proc. Natl. Acad. Sci. U. S. A. 95 (1998) 13018-13023.

[17] S. Cases, et al., Cloning of DGAT2, a second mammalian diacylglycerol acyltransferase, and related family members, J. Biol. Chem. 276 (2001) 38870-38876.

[18] D.L. Brasaemle, et al., Adipose differentiation-related protein is an ubiquitously expressed lipid storage droplet-associated protein, J. Lipid Res. 38 (1997) 2249-2263.

[19] V. Subramanian, et al., Perilipin A mediates the reversible binding of CGI-58 to lipid droplets in 3T3-L1 adipocytes, J. Biol. Chem. 279 (2004) 42062-42071.

[20] C. Londos, D.L. Brasaemle, C.J. Schultz, J.P. Segrest, A.R. Kimmel, Perilipins, ADRP, and other proteins that associate with intracellular neutral lipid droplets in animal cells, Semin. Cell Dev. Biol. 10 (1999) 51-58.

[21] D.L. Brasaemle, G. Dolios, L. Shapiro, R. Wang, Proteomic analysis of proteins associated with lipid droplets of basal and lipolytically stimulated 3T3-L1 adipocytes, J. Biol. Chem. 279 (2004) 46835-46842.

[22] A. Pol, et al., A caveolin dominant negative mutant associates with lipid bodies and induces intracellular cholesterol imbalance, J. Cell Biol. 152 (2001) 1057-1070.

[23] A.G. Ostermeyer, et al., Accumulation of caveolin in the endoplasmic reticulum redirects the protein to lipid storage droplets, J. Cell Biol. 152 (2001) 1071-1078.

[24] D.L. Brasaemle, Thematic review series: adipocyte biology. The perilipin family of structural lipid droplet proteins: stabilization of lipid droplets and control of lipolysis, J. Lipid Res. 48 (2007) 2547-2559.

[25] A.R. Kimmel, D.L. Brasaemle, M. McAndrews-Hill, C. Sztalryd, C. Londos, Adoption of PERILIPIN as a unifying nomenclature for the mammalian PAT-family of intracellular lipid storage droplet proteins, J. Lipid Res. 51 (2010) 468-471.

[26] A.R. Kimmel, C. Sztalryd, The perilipins: major cytosolic lipid droplet-associated proteins and their roles in cellular lipid storage, mobilization, and systemic homeostasis*, Annu. Rev. Nutr. 36 (2016) 471-509.

[27] C. Londos, C. Sztalryd, J.T. Tansey, A.R. Kimmel, Role of PAT proteins in lipid metabolism, Biochimie 87 (2005) 45-49.

[28] J.A. Olzmann, P. Carvalho, Dynamics and functions of lipid droplets, Nat. Rev. Mol. Cell Biol. 20 (2019) 137-155.

[29] A.R. Thiam, R.V. Farese, T.C. Walther, The biophysics and cell biology of lipid droplets, Nat. Rev. Mol. Cell Biol. 14 (2013) 775-786.

[30] R.V. Farese, T.C. Walther, Lipid droplets finally get a little R-E-S-P-E-C-T, Cell 139 (2009) 855-860.

[31] M.J. Gerl, et al., Machine learning of human plasma lipidomes for obesity estimation in a large population cohort, PLoS Biol. 17 (2019) e3000443.

Robin W. Klemm*

Department of Physiology, Anatomy and Genetics, University of Oxford,

United Kingdom

E-mail address: robin.klemm@dpag.ox.ac.uk.

Elina Ikonen ${ }^{\mathrm{a}, \mathrm{b}}$

a Stem Cells and Metabolism Research Program and Dept. of Anatomy, Faculty of Medicine, University of Helsinki, Finland

${ }^{\mathrm{b}}$ Minerva Foundation Institute for Medical Research, Helsinki, Finland 